\title{
The effects of irisin on the rat thoracic aorta: a histological study
}

\author{
R. Altaweel ${ }^{1}$, A. Shatarat ${ }^{1}$, D. Badran ${ }^{1}$, N.M. Abu Tarboush ${ }^{2}$ (I) \\ ${ }^{1}$ Anatomy and Histology Department, School of Medicine, The University of Jordan, Amman, Jordan \\ 2Biochemistry and Physiology Department, School of Medicine, The University of Jordan, Amman, Jordan
}

[Received: 14 July 2021; Accepted: 11 October 2021; Early publication date: 21 October 2021]

Background: Irisin, a polypeptide hormone that is released from skeletal muscle in response to exercise, has been found to improve endothelial functions, protect against endothelial injuries and change blood pressure, which also affected blood vessels. The aim of this study was to assess the histological changes of the rat thoracic aorta in response to irisin injection.

Materials and methods: Twenty-four rats were used. They were divided into two groups: the control group without irisin injection and the irisin-injected group subdivided into three subgroups treated with different irisin concentrations (20, 40 and $160 \mathrm{nM}$, respectively) twice a week for 4 weeks. The control group and irisin-treated subgroups consisted of 6 rats each. After 4 weeks all rats were sacrificed, and the descending thoracic aorta was treated for histological evaluation. Sections were stained with haematoxylin and eosin and orcein stains. Morphometric measurement included: intima-media thickness, number of elastic lamellae and number of smooth muscle cells' nuclei.

Results: Histological study showed that intraperitoneal injection of different concentrations of irisin (20,40 and $160 \mathrm{nM})$ in rats increased intima-media thickness, the number of smooth muscle cell's nuclei and the number of elastic lamellae in media layer of the thoracic aorta in a dose-dependent manner.

Conclusions: Irisin significantly affected the morphology of the wall of the rat thoracic aorta indicating a role of irisin in influencing the growth factors of the thoracic aorta walls and activating smooth muscle cells in the thoracic aorta layers. (Folia Morphol 2022; 81, 4: 923-930)

Key words: irisin, hypertension, blood vessels, thoracic aorta, haematoxylin and eosin, orcein stain, morphometric measurement

\section{INTRODUCTION}

The aorta is the largest artery in the body and carries blood from the heart to the major vessels. It is an elastic artery with a high proportion of elastic fibres in their tunics, especially in tunica media. Like all other arteries, aorta is composed of 3 distinct layers: inner intimal layer, thick middle layer and outer adventitial layer. Each layer is separated from the other layer by an elastic lamina; the internal elastic lamina separates the tunica intima and media while the external elastic lamina separates the tunica media and adventitia [21]. The middle and thickest tunica media is composed mainly of smooth muscle cells (SMCs) and extracellular matrix (elastic fibres and col-

Address for correspondence: Ass. Prof. N.M. Abu Tarboush, Biochemistry and Physiology Department, School of Medicine, The University of Jordan, Queen Rania Street, 11942 Amman, Jordan, e-mail: natarboush@ju.edu.jo

This article is available in open access under Creative Common Attribution-Non-Commercial-No Derivatives 4.0 International (CC BY-NC-ND 4.0) license, allowing to download articles and share them with others as long as they credit the authors and the publisher, but without permission to change them in any way or use them commercially. 
lagen). The vascular smooth muscle cells (VSMCs) are responsible of contraction, production and secretion of collagen and elastic fibres [16]. The elastic fibres in tunica media are arranged in a concentric lamella, these fibres are responsible for distension, recoiling and normalising blood pressure in the aortic wall during pumping [27-29].

It is known that the thickness and numbers of elastic lamellae depend on blood pressure and age. Age increases the arterial diameter, the thickness of intima and media layers, the number of sub-endothelial cells, and the medial calcifications in elastic lamellae [13]. However, it has also been shown that with age there is a decrease in SMCs in tunica media, an increase in the distance between elastic lamellae, elastic fibres' fragmentation and an extreme deposition of collagen $[6,9,13,31]$. Another age-related change has also been reported in tunica intima and adventitia including thickening of these layers due to fibrosis [1].

The second factor affecting the aorta is blood pressure. It has been noted that there is an increase in the number and thickness of lamellae in hypertensive patients and rats $[17,29]$. Under normal conditions, VSMCs are highly differentiated, have poor activity and exhibit low growth levels. On the other hand, under stress and pathological conditions (i.e. hypertension), VSMCs exhibit uncontrolled proliferation which leads to dedifferentiation and accumulation of SMCs in blood vessels' walls causing media thickening and vascular stiffness [25]. Stiffness occurs as a result of aortic elastin fibres fatigue and fragmentation [3]. Thus, hypertension seems to induce ultrastructural changes in the aorta. These changes include increase in the aortic wall, media thickness, elastic lamellae number and thickness [17]. Proliferation of adventitial vasa vasorum was also found to occur due to hypertension [18].

Irisin, a recently discovered myokine, which is released as a result of proteolytic activity of FNDC5 from skeletal muscles to the blood stream in response to exercise [4], has been found to have beneficial effects on regulation of the cardiovascular functions [32], enhance endothelial repair [33], protect against endothelial injury and improve atherosclerosis [15]. Moreover, irisin has been found to relax rat and mouse mesenteric arteries through endothelium dependent and endothelium-independent mechanisms [10, 14, 30, 32].

In this study, we hypothesized that irisin has structural effects on the thoracic aorta. Therefore, we aimed to study the histological changes of the wall of the thoracic aorta in rats in response to irisin injection.

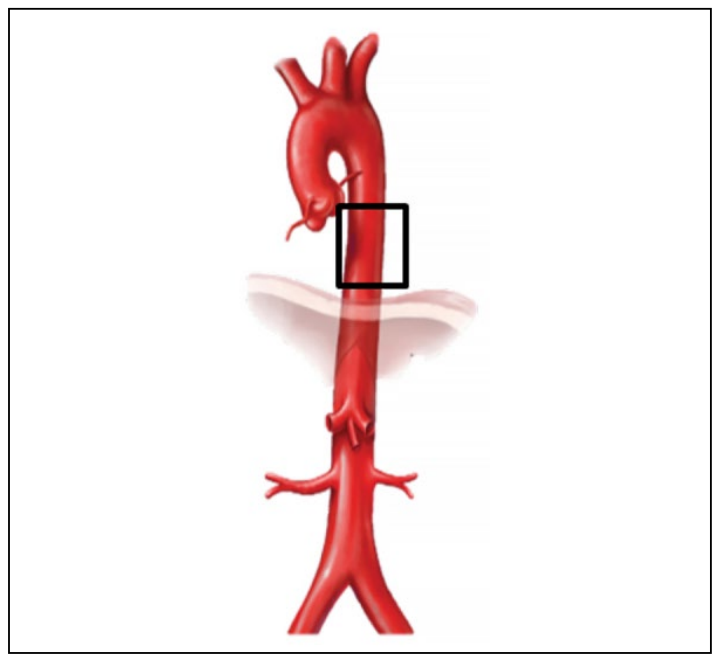

Figure 1. A picture showing the place from where the thoracic aorta has been taken for staining (bold square, cut area)

\section{MATERIALS AND METHODS}

\section{Materials}

Recombinant irisin (human, rat, mouse, canine) was obtained from Phoenix Pharmaceuticals, CA, USA (purity $\geq 95 \%$, molecular weight $\sim 13 \mathrm{KDa}$ ). Irisin was dissolved in dimenthyl sulfoxide (DMSO) (the final DMSO concentration in the diluted working solution was $0.05 \%$ ). Orcein and haematoxylin and eosin (H\&E) were obtained from SIGMA-ALDRICH, USA.

\section{Animals}

Twenty-four albino rats (120-230 g) were obtained from Jordan University of Science and Technology animal's house. All experiments have been performed on 5-7-week-old rats. All rats were maintained in a standard condition (temperature of $20^{\circ} \mathrm{C}$ ) on a $12: 12$-h light-dark cycle and fed a standard chow diet with a free access to water at the University of Jordan animal house. All experiments were approved by the university ethics committee for animal studies. Animals were randomly divided into two main groups: control group $(n=6)$ and experimental or irisin-injected group which was further subdivided into three subgroups $(n=18$, 6 in each group) according to the concentration of irisin injected into each rat $-20,40$ and $160 \mathrm{nM}$, respectively or $1.3 \mathrm{mg} / \mathrm{kg}, 2.6 \mathrm{mg} / \mathrm{kg}$, and $10.2 \mathrm{mg} / \mathrm{kg}$, respectively. Rats were injected with irisin twice a week intraperitoneally for 4 weeks. After 4 weeks, all rats were sacrificed by cervical dislocation, and the descending thoracic aorta was excised (Fig. 1) 
Table 1. Number of sections for haematoxylin and eosin and orcein stains per group

\begin{tabular}{lcc}
\hline Groups & Ring section & Longitudinal section \\
\hline Control & 12 & 12 \\
$20 \mathrm{nM}$ & 12 & 12 \\
$40 \mathrm{nM}$ & 12 & 12 \\
$160 \mathrm{~nm}$ & 12 & 12 \\
\hline
\end{tabular}

and immersed in fixative (formaldehyde 10\%) for histological evaluation. In a separate experiment, to eliminate the placebo effect, rats $(n=4)$ were injected with DMSO (0.05\%). Then the thoracic aorta was taken for histological study. The results showed no histological changes in the rat thoracic aortic sections. Note: food consumption and rats' weight were measured prior and after every week of injection using a digital balance.

\section{Light microscopy}

Rats' thoracic aortae were immersed in $10 \%$ neutral-buffered formalin solution, and kept 48 hours in the fixative solution at room temperature. The samples were routinely processed for paraffin sections. Sections of $5 \mu \mathrm{m}$ thickness were mounted on glass slides and stained with H\&E for general tissue morphology and orcein stain for elastic fibres detection. From each rat multiple sections of thoracic aorta were obtained (two ring and two longitudinal sections), refer to Table 1. The staining procedure for $\mathrm{H} \& \mathrm{E}$ was done according to leicabiosystems/staining overview guide [12]. The sections were stained with newly prepared orcein stain (where $1 \mathrm{~g}$ orcein was dissolved in $100 \mathrm{~mL} 70 \%$ ethanol and $1 \mathrm{~mL} 25 \% \mathrm{HCl}$ was added) according to Unna [26] and as described by Romeis (1989) [20].

The prepared sections were examined and photographed using LEICA EC3 inverted light microscope equipped with LEICA CH, 9435, Switzerland camera connected to computer with LEICA Application Suite E2 version 1.8.0 software (LAS EZ).

\section{Morphometric measurements}

The stained sections with $\mathrm{H} \& \mathrm{E}$ and orcein were examined using LEICA Application Suite E2 version 1.8.0 software (LAS EZ) and ImageJ software. Twenty four preparations in each stain were subjective to quantitative studies: intima-media thickness (IMT), number of elastic lamellae and number of SMCs' nuclei.
For the IMT measurements, the whole intima and media layer thicknesses were included in the H\&E and orcein staining. The thickness was taken by drawing a line from the intimal layer (marked by luminal surface of intima) to the end of medial layer (marked by the external elastic lamina). Two lines were drawn from the luminal surface of intima to the external elastic lamina. The IMT was measured by taking the average of the two lines (in both ring and longitudinal sections) for each rat in each group and then the average of IMT of all rats was calculated and taken as final measurement. The measurements were calculated in scale bar $=50 \mu \mathrm{m}$. The number of elastic lamellae was measured manually by counting the elastic lamellae layers in the orcein stained segments. The total number of elastic lamellae was measured by taking the average of all elastic lamellae numbers from all segments of the same group. The number of SMCs nuclei was measured by using ImageJ software where the section picture was opened and adjusted in a suitable threshold to be analysed and counted.

\section{Statistical analysis}

Statistical analyses were performed using GraphPad Prism version 5 for Windows (GraphPad Software, CA, USA). Statistical comparisons were obtained by one-way analysis of variance (ANOVA) followed by Dunnett's multiple comparison test and Student paired $t$ test. Values of $p<0.05$ were considered statistically significant. The quantitative data were examined by LEICA Application Suite E2 version 1.8.0 software (LAS EZ) and ImageJ software.

\section{RESULTS}

General microscopic structure of rat thoracic aorta in control and experimental groups, as shown by using H\&E staining

Haematoxylin and eosin sections of the rat thoracic aorta of the control group showed normal histological features for all layers. Tunica intima appeared as a continuous layer of endothelial cells with darkly stained nuclei. This layer was lying on an internal elastic lamina which appeared as a continuous lamina. Tunica media showed normal, wavy multiple elastic lamellae which were concentrically arranged and normal spindle shaped SMCs were seen between the concentric elastic lamellae. An external elastic lamina was also observed close to tunica adventitia as normal continuous lamina. Typical normal adventitial layer containing fibroblast, collagen and vasa vasorum was also clearly seen (Fig. 2A). 


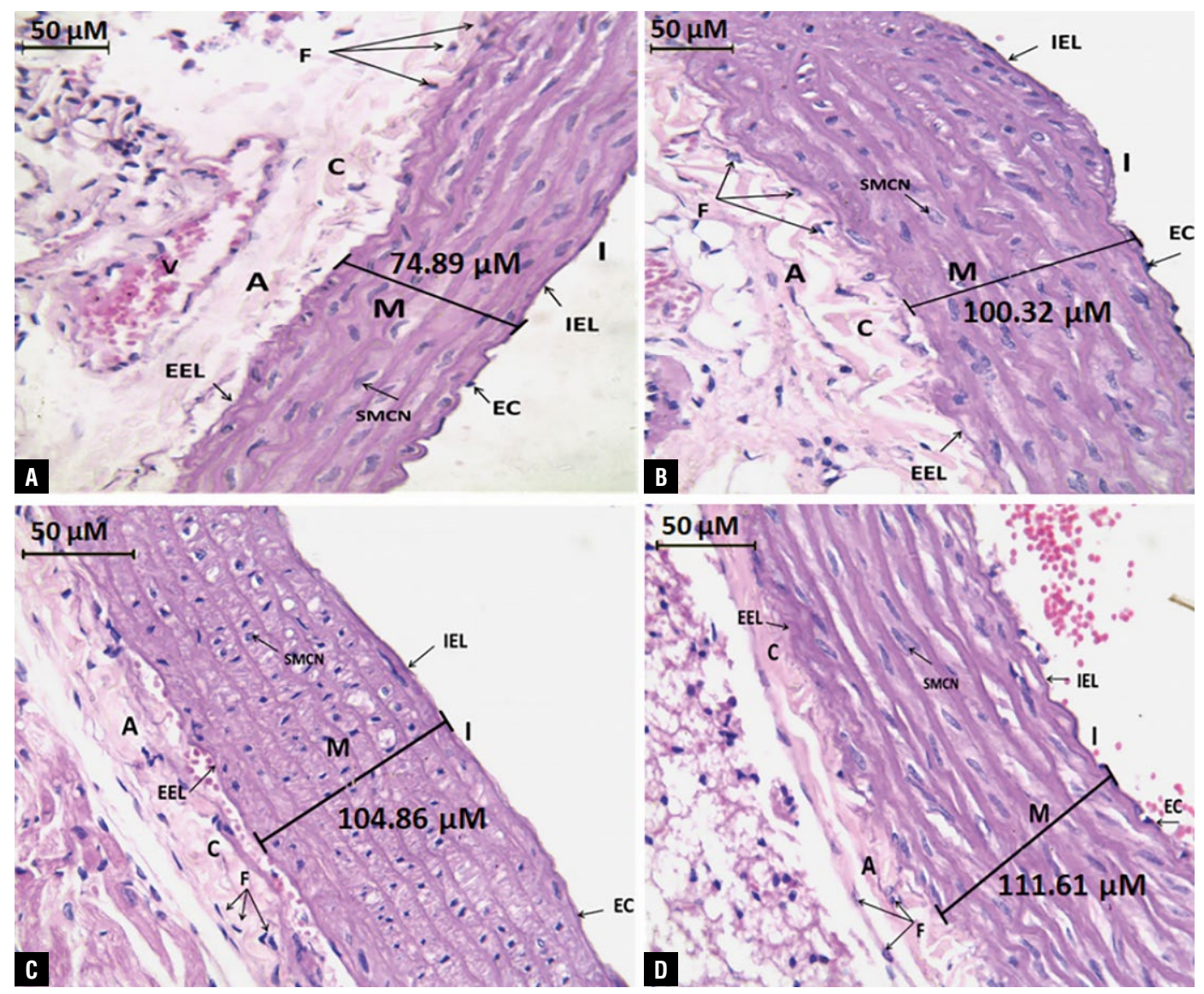

Figure 2. Histological sections of rat thoracic aorta wall stained with haematoxylin and eosin $(\times 400)$; $A$. Endothelial cells (EC, arrowhead) line the tunica intima (I), tunica media (M), and tunica adventitia (A) of the control group; B. An increase in the intima-media thickness (IMT), lamellae of elastic fibres and smooth muscle cell nuclei in irisin-injected group 1; C. An increase in the IMT, lamellae of elastic fibres and hyperplasia of the smooth muscle cell nuclei in irisin-injected group 2 (a longitudinal section); D. An increase in the IMT, lamellae of elastic fibres and hyperplasia of the smooth muscle cell nuclei in irisin-injected group 3. Collagen $(\mathrm{C})$, fibroblast cells $(\mathrm{F}$, arrows) and blood vessels called vasa vasorum (V). The internal elastic lamina (IEL) separates the tunica intima and media, and the external elastic lamina (EEL) separates the tunica media and adventitia, small muscle cell nucleus (SMCN).
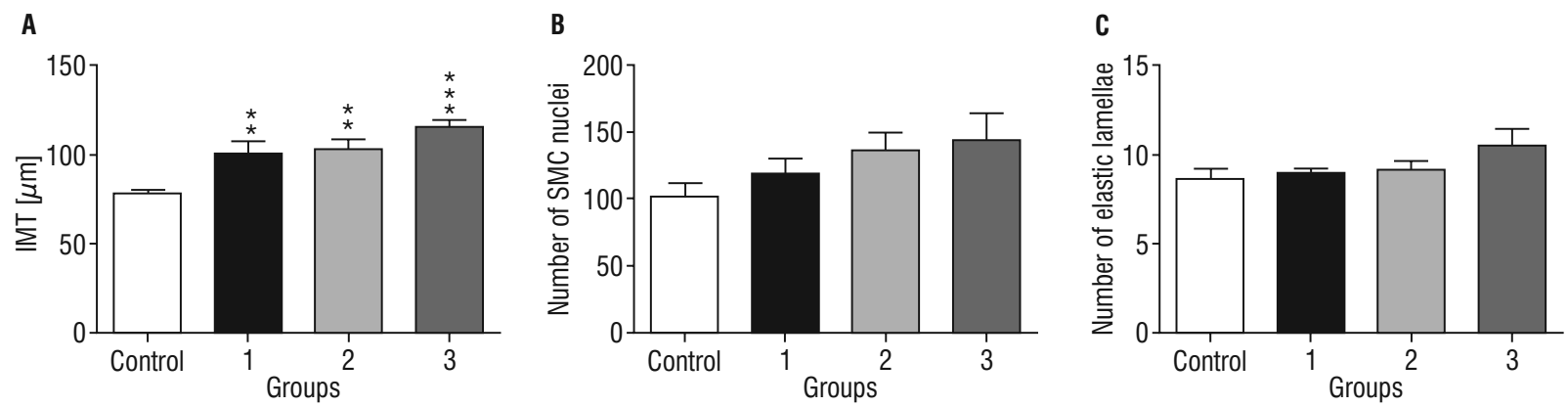

Figure 3. Aortic media components in different groups; A. The changes in intima-media thickness (IMT) between the control group and different treatment groups of irisin; B. The number of smooth muscle cell (SMC) nuclei in the control group and different treatment groups of irisin; C. The number of elastic lamellae in the control group and different treatment groups of irisin. Each bar represents the mean \pm standard error of mean; ${ }^{* *} \mathrm{p}<0.005 \mathrm{vs}$. control, ${ }^{* * *} \mathrm{p}<0.0001 \mathrm{vs}$. control (one-way ANOVA followed by Dunnett's multiple comparison test, each group $\mathrm{n}=6)$. Groups 1, 2, and 3 received $20 \mathrm{nM}(1.3 \mu \mathrm{g} / \mathrm{kg}), 40 \mathrm{nM}(2.6 \mu \mathrm{g} / \mathrm{kg})$, and $60 \mathrm{nM}(10.2 \mu \mathrm{g} / \mathrm{kg})$, respectively.

Sections of the rat thoracic aorta in all irisin-injected groups $(20,40$ and $160 \mathrm{nM})$ showed dose-dependent increase in the IMT compared to that of the control group (from $78.3 \mu \mathrm{m}$ in the 
Table 2. Measurement of the aortic media components in different groups

\begin{tabular}{|c|c|c|c|c|}
\hline & Control group & $\begin{array}{c}\text { Irisin-injected group } 1 \\
(1.3 \mu \mathrm{g} / \mathrm{kg})\end{array}$ & $\begin{array}{l}\text { Irisin-injected group } 2 \\
(2.6 \mu \mathrm{g} / \mathrm{kg})\end{array}$ & $\begin{array}{c}\text { Irisin-injected group } 3 \\
(10 \mu \mathrm{g} / \mathrm{kg})\end{array}$ \\
\hline Intima-media thickness $[\mu \mathrm{m}]$ & $78.3 \pm 1.6$ & $100.5 \pm 6.3^{* *}$ & $103.2 \pm 5.3^{* *}$ & $115.4 \pm 3.9 * * *$ \\
\hline Number of elastic lamellae & $8.6 \pm 0.55$ & $9 \pm 0.25$ & $9.1 \pm 0.47$ & $10.5 \pm 0.95$ \\
\hline Number of smooth muscle cell nuclei & $102 \pm 11$ & $119 \pm 11.5$ & $136 \pm 13.4$ & $144 \pm 20.2$ \\
\hline
\end{tabular}

Significant at ${ }^{* *} p<0.005$ vs. control; ${ }^{* * *} p<0.0001$ vs. control (one-way ANOVA followed by Dunnett's multiple comparison test), each group ( $n=6$ ).

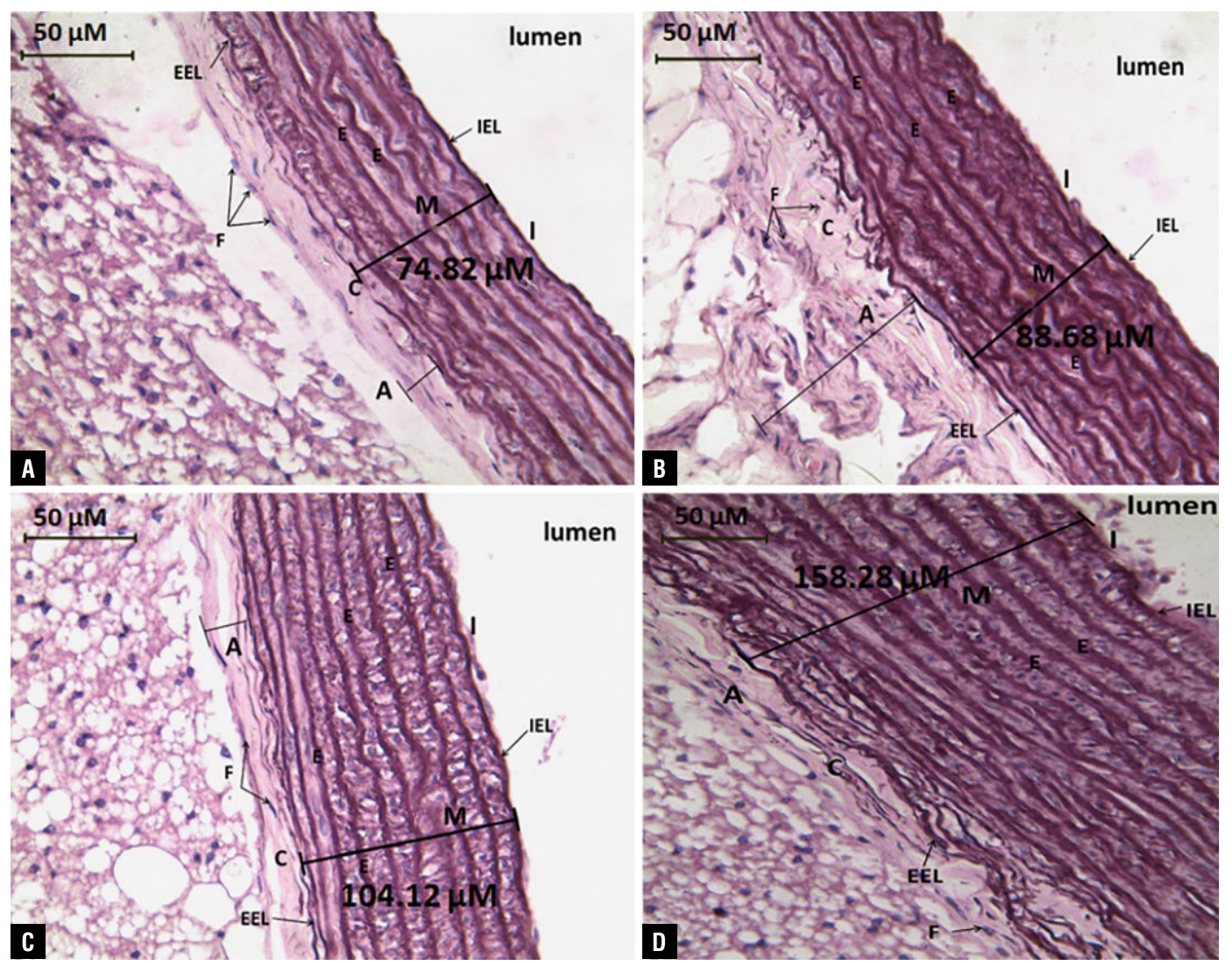

Figure 4. Histological sections of rat thoracic aorta wall stained with orcein stain $(\times 400)$. Lamellae of elastic fibres $(E)$ are shown for the control group (A), irisin-injected group 1 (B), irisin-injected group 2 (C), and irisin-injected group 3 (D). Note the number of elastic lamellae in the experimental groups in comparison to control group; I — tunica intima; $\mathrm{M}$ - tunica media; $\mathrm{A}$ — tunica adventitia; $\mathrm{C}$ - collagen; F — fibroblast; IEL — internal elastic lamina; EEL — external elastic lamina.

control group, to $100.5,103.2$ and $115.4 \mu \mathrm{m}$, in irisin-injected rats, respectively); the increase in IMT was most evident in group 3 (160 nM). The increase was in the number of both elastic lamellae and SMCs. An increase in the number of SMCs nuclei also was noticed (from $102 \pm 20$ in the control group to $119 \pm 33,136 \pm 34$ and $144 \pm 20$ in irisin-injected rats, respectively) and it was more evident in group 3 (160 nM), but it was not significant (Figs. 2B-D; 3; Table 2).

\section{Histological structure of rat thoracic aorta in control and experimental groups, as shown by orcein staining}

The orcein stained sections of the rat thoracic aorta of the control group showed normal histological features for all layers. Tunica media showed normal multiple elastic lamellae (Fig. 4A). The orcein stained sections showed dose-dependent increase in the elastic lamellae in irisin-injected groups (20, 40 and $160 \mathrm{nM})$ compared to that of the control group (8.6 layers in control rats). The 

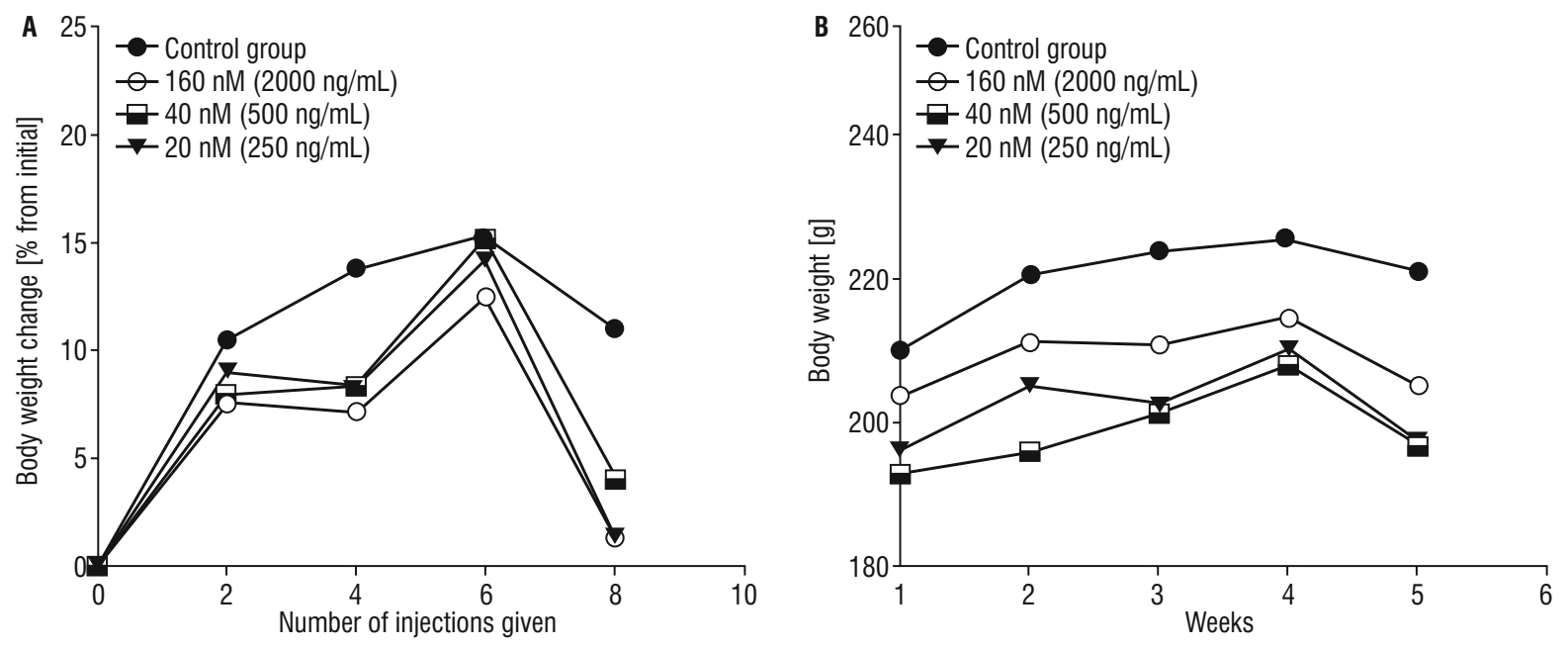

Figure 5. Body weight changes in control and experimental groups; A. A line graph showing body weights change (\% from initial) in rats injected with irisin and without injection (control); B. A line graph showing changes in body weight (g) for 6 animals in each group. The change in rats' weight is significant. One-way ANOVA followed by Dunnett's multiple comparison test, each group $n=6$.

increase in elastic lamellae was more evident in group 3 (dose $160 \mathrm{nM}$ ) with 10.5 layers. The increase was in the number of elastic lamellae (Figs. 3; 4B-D; Table 2).

\section{Body weight change and food intake results}

Irisin injection did not affect food consumption in the rats. Body weights decreased in irisin-injected groups and this change in rats' body weights were statistically significant (Fig. 5). Significance has been shown in the $160 \mathrm{nM}$ injected groups vs. control $(\mathrm{p}<0.05)$ and in $30 \mathrm{nM}$ and $40 \mathrm{nM}$ injected groups vs. control $(p<0.001)$.

\section{DISCUSSION}

The present study investigated the possible histological changes of the wall of the thoracic aorta in rats injected with irisin intraperitoneally (in vivo). The results of this study have demonstrated for the first time an increase in the IMT and in the number of both SMCs nuclei and elastic laminae in the wall of rat thoracic aorta injected with irisin compared to non-injected (control) group under light microscope using H\&E and orcein dyes.

Blood vessels are responsive to two major factors that can affect their development in healthy and pathological conditions; these factors are age and blood pressure. Age for example, increases the arterial wall diameter due to an increase in the thickness of the intima, media, and the elastic lamellae [13]. While high blood pressure causes changes in the walls of blood vessels, which makes them more vulnerable to develop cardiovascular diseases [13]. Elastin fibres of the aorta in hypertension become fatigued and fragmented, which results in stiff aorta [3]. Thus, hypertension seems to induce ultrastructural changes in the aorta. These changes include increase in the aortic wall, media thickness, elastic lamellae number and thickness [17]. Proliferation of adventitial vasa vasorum has also been found to occur due to hypertension [18]. All the previously mentioned changes in response to increase in blood pressure make the heart work harder which may lead to cardiac hypertrophy [1].

Recently, irisin, an exercise-mediated polypeptide has attracted considerable attention due to its involvement in the treatment of cardiovascular and metabolic diseases [5] and its effect in lowering blood pressure [7].

A recent study has found that irisin lowered blood pressure in spontaneously hypertensive rats [7] indicating the possible involvement of irisin in the regulation of blood pressure. It is known that blood pressure can significantly modulate the structure of the blood vessels where an increase in the number and thickness of elastic lamellae in hypertensive patients and rats has been reported $[17,29]$. The present study showed, by using light microscope and two different stains (H\&E and orcein for elastic tissue), the changes in the wall of thoracic aorta in response to irisin injection. The results showed a statistically significant dose dependent increase in IMT in irisin injected groups. Also an increase 
in the number of elastic fibres and lamellar layers and hyperplasia of SMCs nuclei and condensation to their nuclei were also noticed in the rat thoracic aorta, where it was more prominent in rats who received the highest irisin dose $160 \mathrm{nM}$.

The increase in IMT in irisin-injected groups can be explained by the fact that irisin may have stimulated the endothelial cells of the rat thoracic aorta to produce growth factors which in turn have stimulated the growth of VSMCs and hence increased the production of elastic fibres [22, 23]. It is well known that VSMCs are responsible for the secretion and production of collagen and elastic fibres in blood vessels [24]. Therefore, any changes in the number of elastic fibres should be attributed to the VSMCs. It has been reported that endothelial cells have been involved in the regulation of VSMCs through their secretion of growth factors such as PDGF-B $[2,11]$.

It is worth mentioning that the morphological changes induced by irisin in the present study were not due to an increase of rat's age. The whole experiment lasted for a month which is not a lengthy period relative to the rat's lifespan which is estimated to be approximately 3 years [19]. In addition, there were no morphological changes in the control group indicating that the morphological changes in the wall of the rat thoracic aorta were most likely attributed to irisin.

It is also important to note that after irisin injection, rats did not show any notable changes in food or water intake. On the other hand, rats injected with irisin showed decreased body weights, which could be explained by the fact that irisin plays an important role in fat metabolism [8], which may be crucial for the treatment of obesity in the future.

\section{CONCLUSIONS}

This study has shown that irisin significantly affects the morphology of the wall of the rat thoracic aorta by increasing its IMT, number of SMCs nuclei and the number of elastic lamellae in tunica media. These results indicate that irisin may influence the growth factors of the thoracic aorta walls and activate SMCs in the thoracic aorta layers.

\section{Acknowledgements}

This work was supported by a grant from the Deanship of Academic Research - The University of Jordan. We thank Mrs. Duaa Al Qattan for laboratory technical assistance, and Mr. Ahmed from the faculty of biology for his contribution in sections imaging.

\section{Conflict of interest: None declared}

\section{REFERENCES}

1. Abu-Dief EE, Abdelrahim EA, Abdelrahim KM. Histological Modifications Aging Aorta in Male Albino Rat. J Cytol Histol. 2016; 7(2): 6, doi: 10.4172/2157-7099.1000408.

2. Andrae J, Gallini R, Betsholtz C. Role of platelet-derived growth factors in physiology and medicine. Genes Dev. 2008; 22(10): 1276-1312, doi: 10.1101/gad.1653708, indexed in Pubmed: 18483217.

3. Benetos A, Adamopoulos C, Bureau JM, et al. Determinants of accelerated progression of arterial stiffness in normotensive subjects and in treated hypertensive subjects over a 6-year period. Circulation. 2002; 105(10): 1202-1207, doi: 10.1161/hc1002.105135, indexed in Pubmed: 11889014.

4. Boström P, Wu J, Jedrychowski MP, et al. A PGC1- $\alpha$-dependent myokine that drives brown-fat-like development of white fat and thermogenesis. Nature. 2012; 481(7382): 463-468, doi: 10.1038/nature10777, indexed in Pubmed: 22237023.

5. Chen N, Li Q, Liu J, et al. Irisin, an exercise-induced myokine as a metabolic regulator: an updated narrative review. Diabetes Metab Res Rev. 2016; 32(1): 51-59, doi: 10.1002/ dmrr.2660, indexed in Pubmed: 25952527.

6. Fritze $O$, Romero B, Schleicher M, et al. Age-related changes in the elastic tissue of the human aorta. J Vasc Res. 2012; 49(1): 77-86, doi: 10.1159/000331278, indexed in Pubmed: 22105095.

7. Fu J, Han Yu, Wang J, et al. Irisin lowers blood pressure by improvement of endothelial dysfunction via AMPKAkt-eNOS-NO pathway in the spontaneously hypertensive rat. J Am Heart Assoc. 2016; 5(11), doi: 10.1161/ JAHA.116.003433, indexed in Pubmed: 27912206.

8. Fukushima $Y$, Kurose $S$, Shinno $H$, et al. Effects of body weight reduction on serum irisin and metabolic parameters in obese subjects. Diabetes Metab J. 2016; 40(5): 386-395, doi: 10.4093/dmj.2016.40.5.386, indexed in Pubmed: 27766246.

9. Greenwald SE. Ageing of the conduit arteries. J Pathol. 2007; 211(2): 157-172, doi: 10.1002/path.2101, indexed in Pubmed: 17200940.

10. Han F, Zhang S, Hou N, et al. Irisin improves endothelial function in obese mice through the AMPK-eNOS pathway. Am J Physiol Heart Circ Physiol. 2015; 309(9): H1501-H1508, doi: 10.1152/ajpheart.00443.2015, indexed in Pubmed: 26371167.

11. Hellström $M$, Kalén $M$, Lindahl $P$, et al. Role of PDGF-B and PDGFR-beta in recruitment of vascular smooth muscle cells and pericytes during embryonic blood vessel formation in the mouse. Development. 1999; 126(14): 3047-3055, doi: 10.1242/dev.126.14.3047, indexed in Pubmed: 10375497.

12. https://www.leicabiosystems.com/knowledge-pathway/ he-staining-overview-a-guide-to-best-practices/.

13. Jani B, Rajkumar C. Ageing and vascular ageing. Postgrad Med J. 2006; 82(968): 357-362, doi: 10.1136/ pgmj.2005.036053, indexed in Pubmed: 16754702. 
14. Jiang $M$, Wan F, Wang $F$, et al. Irisin relaxes mouse mesenteric arteries through endothelium-dependent and endothelium-independent mechanisms. Biochem Biophys Res Commun. 2015; 468(4): 832-836, doi: 10.1016/j. bbrc.2015.11.040, indexed in Pubmed: 26582714.

15. Lu J, Xiang G, Liu M, et al. Irisin protects against endothelial injury and ameliorates atherosclerosis in apolipoprotein E-Null diabetic mice. Atherosclerosis. 2015; 243(2): 438-448, doi: 10.1016/j.atherosclerosis.2015.10.020, indexed in Pubmed: 26520898.

16. Majesky MW, Dong XR, Regan JN, et al. Vascular smooth muscle progenitor cells: building and repairing blood vessels. Circ Res. 2011; 108(3): 365-377, doi: 10.1161/ CIRCRESAHA. 110.223800, indexed in Pubmed: 21293008.

17. Nasiri Z, Sameni HR, Vakili A, et al. Dietary saffron reduced the blood pressure and prevented remodeling of the aorta in L-NAME-induced hypertensive rats. Iranian J Basic Med Sci. 2015; 18(11): 1143.

18. Ogeng'o J, Ongeti $K$, Obimbo $M$, et al. Features of atherosclerosis in the tunica adventitia of coronary and carotid arteries in a black kenyan population. Anat Res Int. 2014; 2014: 456741, doi: $10.1155 / 2014 / 456741$, indexed in Pubmed: 24778879.

19. Quinn R. Comparing rat's to human's age: how old is my rat in people years? Nutrition. 2005; 21(6): 775-777, doi: 10.1016/j.nut.2005.04.002, indexed in Pubmed: 15925305.

20. Romeis B. Mikroskopische Technik. Urban und Schwarzenberg, München 1989.

21. Sandow SL, Gzik DJ, Lee RM. Arterial internal elastic lamina holes: relationship to function? J Anat. 2009; 214(2): 258-266, doi: 10.1111/j.1469-7580.2008.01020.x, indexed in Pubmed: 19207987.

22. Song $H, W u F$, Zhang $Y$, et al. Irisin promotes human umbilical vein endothelial cell proliferation through the ERK signaling pathway and partly suppresses high glucose-induced apoptosis. PLoS One. 2014; 9(10): e110273, doi: 10.1371/ journal.pone.0110273, indexed in Pubmed: 25338001.

23. Song $\mathrm{H}, \mathrm{Xu} \mathrm{J,} \mathrm{Lv} \mathrm{N}$, et al. Irisin reverses platelet derived growth factor-BB-induced vascular smooth muscle cells phenotype modulation through STAT3 signaling pathway.
Biochem Biophys Res Commun. 2016; 479(2): 139-145, doi: 10.1016/j.bbrc.2016.07.052, indexed in Pubmed: 27416763.

24. Tonar Z, Witter K, Krizkova V, et al. Stereological tools for quantitative microscopy of the aortic wall with focus on the abdominal aortic aneurysm. Microscopy: Science, Technology, Applications and education. A Mendez-Vilas J Diaz. 2010: 926-935.

25. Touyz RM, Alves-Lopes R, Rios FJ, et al. Vascular smooth muscle contraction in hypertension. Cardiovasc Res. 2018; 114(4): 529-539, doi: 10.1093/cvr/cvy023, indexed in Pubmed: 29394331.

26. Unna PG. Uber die Taenzersche Farbung des elastischen Gewebes. Monatsh Prakt Dermat. 1891; 11: 365.

27. Wachi H. Role of elastic fibers on cardiovascular disease. J Health Sci. 2011; 57(6): 449-457, doi: 10.1248/jhs.57.449.

28. Wagenseil JE, Ciliberto $\mathrm{CH}$, Knutsen $\mathrm{RH}$, et al. The importance of elastin to aortic development in mice. Am J Physiol Heart Circ Physiol. 2010; 299(2): H257-H264, doi: 10.1152/ ajpheart.00194.2010, indexed in Pubmed: 20495146.

29. Wagenseil JE, Mecham RP. Elastin in large artery stiffness and hypertension. J Cardiovasc Transl Res. 2012; 5(3): 264-273, doi: 10.1007/s12265-012-9349-8, indexed in Pubmed: 22290157.

30. Ye Li, Xu M, Hu M, et al. TRPV4 is involved in irisin-induced endothelium-dependent vasodilation. Biochem Biophys Res Commun. 2018; 495(1): 41-45, doi: 10.1016/j. bbrc.2017.10.160, indexed in Pubmed: 29097199.

31. Yildiz $O$. Vascular smooth muscle and endothelial functions in aging. Ann N Y Acad Sci. 2007; 1100: 353-360, doi: 10.1196/annals.1395.038, indexed in Pubmed: 17460198.

32. Zhang W, Chang L, Zhang C, et al. Central and peripheral irisin differentially regulate blood pressure. Cardiovasc Drugs Ther. 2015; 29(2): 121-127, doi: 10.1007/s10557015-6580-y, indexed in Pubmed: 25820670.

33. Zhu G, Wang J, Song M, et al. Irisin increased the number and improved the function of endothelial progenitor cells in diabetes mellitus mice. J Cardiovasc Pharmacol. 2016; 68(1): 67-73, doi: 10.1097/FJC.0000000000000386, indexed in Pubmed: 27002278. 